\title{
Biodiversité des macrochampignons sauvages comestibles de la forêt humide du Sud-Cameroun
}

Nérée Onguene Awana ${ }^{1}$

Armelle Nadine TCHUdJO TCHUENTE ${ }^{1}$ Thomas W. KUYPER ${ }^{2}$

\author{
${ }^{1}$ Institut de recherche agricole \\ pour le développement (IRAD) \\ Département des forêts, \\ sols et environnement \\ BP 2123, Yaoundé \\ Cameroun
}

\section{${ }^{2}$ University of Wageningen}

Department of Environmental Sciences, Sub-department of Soil Quality PO Box 47

6700AA Wageningen

The Netherlands

\section{Auteur correspondant /} Corresponding author: Nérée Onguene Awana nereeoa678@yahoo.fr

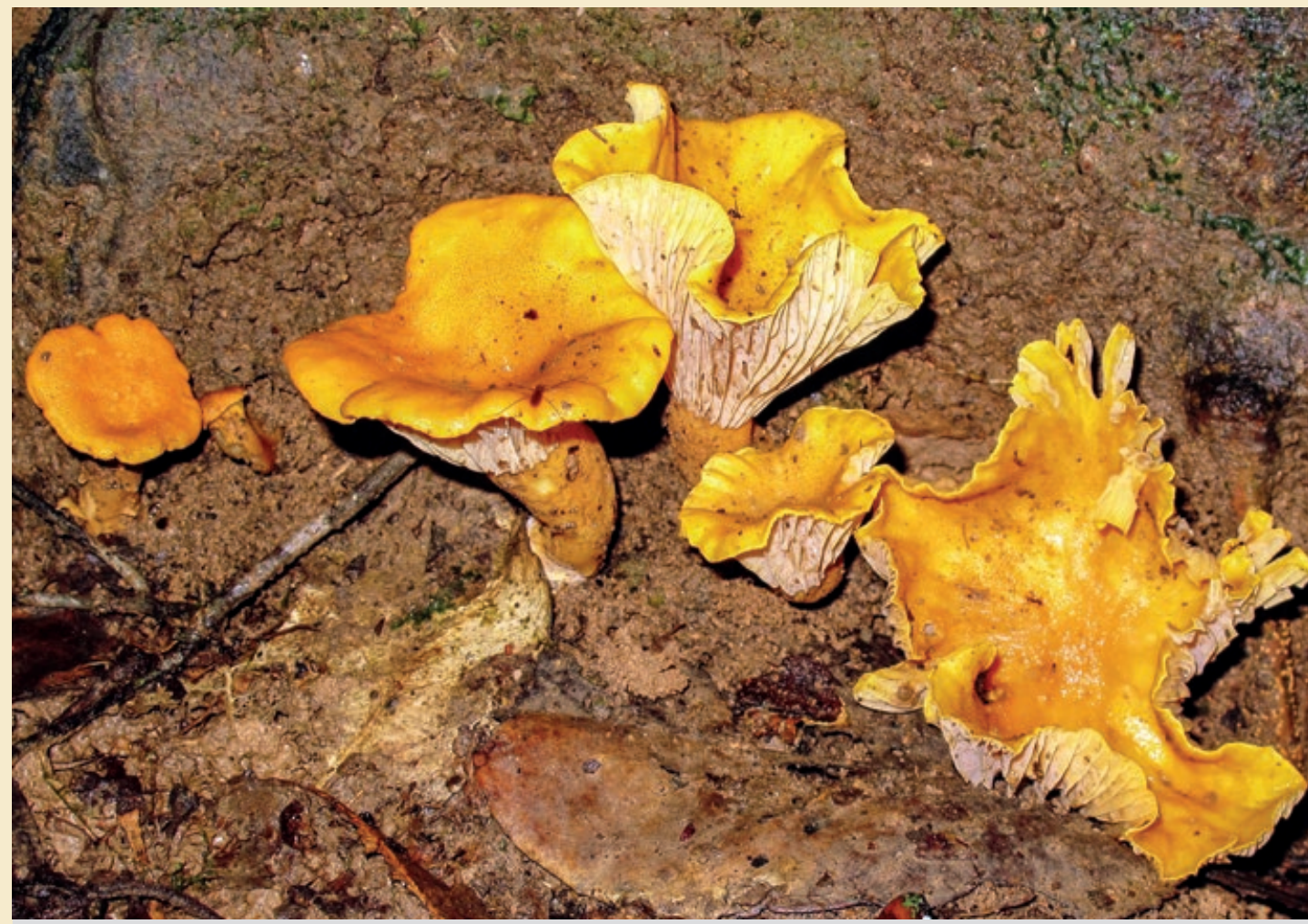

Photo 1.

Cantharellus rufopunctatus (Beeli) Heinem.

Photo N. Onguene Awana.

Doi : 10.19182/bft2018.338.a31679 - Droit d'auteur (c) 2018, Bois et Forêts des Tropiques @ Cirad - Date de soumission : 10 février 2018 ; date d'acceptation : 14 août 2018 ; date de publication : $1^{\text {er }}$ décembre 2018.

\section{Ocirad (6)}

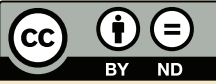

Citer l'article / To cite the article

Onguene Awana N., Tchudjo Tchuente A. N., Kuyper T. W., 2018. Biodiversité des macrochampignons sauvages comestibles de la forêt humide du Sud-Cameroun. Bois et Forêts des Tropiques, $n^{\circ} 338$ : 87-99. Doi : https:// doi.org/10.19182/bft2018.338.a31679 


\section{N. Onguene Awana, \\ A. N. TCHUDJO TCHUENTE, T. W. KUYPER}

\section{ABSTRACT}

\section{Biodiversity of wild edible macrofungi in southern Cameroon's humid forests}

\section{Biodiversité des macrochampignons sauvages comestibles de la forêt humide du Sud-Cameroun}

Pour les communautés de forêts humides du Cameroun - Bantu, pygmées Baka et Bagyeli -, les champignons sauvages comestibles (CSC) ont toujours été considérés comme de substantielles sources alimentaires et médicinales. Cependant, peu d'informations sont disponibles sur la diversité et l'écologie des CSC du Cameroun. Aussi une étude a-t-elle été entreprise pour connaître la diversité et acquérir les connaissances endogènes des principaux champignons sauvages comestibles et médicinaux de la forêt humide de ce pays. À partir d'excursions mycologiques, d'enquêtes structurées et semi-structurées conduites dans une vingtaine de sites de cinq régions du Grand Sud-Cameroun, les espèces de CSC ont été collectées et décrites. Quatre-vingt-quatorze taxons de CSC ont été identifiés, appartenant à 32 familles et 41 genres, dont environ $61,7 \%$ de saprotrophes, 21,3\% de taxons ectomycorhiziens (ECM) et $17 \%$ d'espèces du genre Termitomyces, les plus fréquemment collectées et consommées. Cette forte diversité fongique s'est traduite par un éventail varié d'habitats et de niches écologiques. Les CSC les plus appréciés sont les saprotrophes Armillaria camerounensis et Volvariella volvacea, ainsi que toutes les espèces de Termitomyces et de chanterelles. La plupart des CSC sont collectés pour la consommation. La vingtaine d'espèces de CSC ectomycorhiziens sont symbiotiquement associées aux racines de 13 genres d'essences forestières appartenant aux familles des Cesalpiniaceae et Phyllantaceae. In fine, deux principaux services écosystémiques de production sont fournis par les CSC du Cameroun : l'approvisionnement alimentaire et médicinal, et l'octroi de revenus. Des besoins de recherche s'imposent en faveur de l'étude de la biodiversité des CSC de la forêt humide du Cameroun, et de la valorisation des déchets agricoles pour la culture du champignon de paille V. volvacea.

Mots-clés : Termitomyces, Uapaca, Cesalpiniaceae, ectomycorhizes, Armillaria camerounensis, Volvariella volvacea, saprotrophes, Baka, Bagyeli, Bantu, Cameroun.
The communities living in the humid forests of southern Cameroon - Bantu, and Baka and Bagyeli pygmies - have always considered wild edible fungi (WEF) as important sources of food and medicine. However, little information is available on the diversity and ecology of wild edible fungi in Cameroon. This study was therefore undertaken to investigate and acquire endogenous knowledge on the diversity of the main wild edible and medicinal fungi in Cameroon's humid forests. The fungi species were collected during mycological excursions and described through structured and semi-structured surveys conducted in some twenty sites in five regions of southern Cameroon. Ninety-four WEF taxa were identified, belonging to 32 families and 41 genera. About $61.7 \%$ were saprotrophic taxa, $21.3 \%$ ectomycorrhizal (ECM) and $17 \%$ of the Termitomyces genus, the latter being the commonly collected and consumed. The high diversity of the fungi was reflected in their varied range of habitats and ecological niches. The most prized WEF are the saprotropic Armillaria camerounensis and Volvariella volvacea species, all Termitomyces species and chanterelles. Most of these fungi are collected as food. The twentyodd ectomycorrhizal species develop in symbiosis with the roots of forest tree species of 13 genera in the Cesalpiniaceae and Phyllantaceae families. Overall, wild edible fungi in Cameroon provide two main productive ecosystem services: supplies of food and medicine, and revenues. Research is clearly needed on the biodiversity of WEF and medicinal species in Cameroon's humid forests and on possibilities for composting agricultural waste to grow $V$. volvacea mushrooms.

\section{Keywords: Termitomyces, Uapaca,} Cesalpiniaceae, ectomycorrhizal, Armillaria camerounensis, Volvariella volvacea, saprotrophic, Baka, Bagyeli, Bantu, Cameroon.

\section{RESUMEN}

\section{Biodiversidad de los macrohongos silvestres comestibles del bosque húmedo del sur del Camerún}

Para las comunidades de bosques húmedos del Camerún, Bantu, pigmeos Baka y Bagyeli, los hongos silvestres comestibles (HSC) desde siempre se han considerado como importantes fuentes alimenticias y medicinales. Sin embargo, hay poca información disponible sobre la diversidad y ecología de los HSC del Camerún. Se ha llevado a cabo un estudio para conocer su diversidad y adquirir los conocimientos endógenos de los principales hongos silvestres comestibles y medicinales del bosque húmedo de este país. Mediante excursiones micológicas, e investigaciones estructuradas y semiestructuradas llevadas a cabo en una veintena de lugares de cinco regiones del gran sur del Camerún, se recogieron y describieron las especies de HSC. Se identificaron 94 taxones de HSC, pertenecientes a 32 familias y 41 géneros, cerca de un $61,7 \%$ de los cuales son saprófagos, 21,3 \% son taxones ectomicorrícicos (ECM) y $17 \%$ especies del género Termitomyces, los recogidos y consumidos con mayor frecuencia. Esta gran diversidad fúngica se traduce en un abanico variado de hábitats y nichos ecológicos. Los HSC más apreciados son los saprófagos Armillaria camerounensis y Volvariella volvacea, así como todas las especies de Termitomyces y de rebozuelos. La mayor parte de HSC se recogen para el consumo. La veintena de especies de HSC ectomicorrícicas están simbióticamente asociadas a las raíces de 13 géneros de especies forestales pertenecientes a las familias de las Cesalpiniaceae y Phyllantaceae. En conclusión, los HSC del Camerún proporcionan dos servicios ecosistémicos principales de producción: el aprovisionamiento alimenticio y medicinal, y la obtención de ingresos. Resulta necesario investigar sobre la biodiversidad y las propiedades medicinales de los HSC del bosque húmedo del Camerún, y sobre la valorización de los residuos agrícolas para el cultivo de la paja seta, $V$. volvacea.

Palabras clave: Termitomyces, Uapaca, Cesalpiniaceae, ectomicorrizas, Armillaria camerounensis, Volvariella volvacea, saprófagos, Baka, Bagyeli, Bantu, Camerún. 


\section{Introduction}

Depuis des millénaires en Chine et chez les Romains, et depuis plusieurs siècles chez les communautés sédentaires Bantou et les peuples autochtones nomades des forêts humides d'Afrique centrale, les champignons sauvages comestibles (CSC) constituent d'excellentes ressources alimentaires primaires, surtout en périodes de soudure, et secondairement médicinales (Boa, 2006 ; Eyi et al., 2014). Dans ce contexte, la collecte, la consommation et l'utilisation des champignons sous-tendent des pratiques traditionnelles et culturelles très anciennes (Härkönen et al., 1994 ; Yorou et De Kesel, 2002 ; Boa, 2006 ; Teke et al., 2018). Cependant, les communautés de macrochampignons sauvages comestibles du Cameroun, qui représentent une des plus importantes sources de biodiversité alimentaire, restent faiblement valorisées et leurs connaissances peu capitalisées.

En Afrique subsaharienne, environ 551 espèces de champignons sauvages comestibles ont été identifiées (Boa, 2006). Une revue désormais ancienne a été publiée sur les usages et l'importance des CSC pour les régimes alimentaires des populations locales d'Afrique subsaharienne (Rammeloo et Walleyn, 1993). Plusieurs études ont rapporté la quantité de champignons sauvages consommés en Tanzanie et à travers le monde (Rammeloo et Walleyn, 1993 ; Härkönen et al., 1994, 2003 ; Mueller et al., 2007). L'importance et les usages des CSC ont été décrits pour la communauté Yoruba au Nigeria, les pygmées Aka et Bofi de la République centrafricaine (Heim, 1942 ; Oso, 1975, 1977 ; Malaisse et al., 2008), les Bantu et les Bagyeli du Cameroun (Dijk et al., 2003), les Nagots du Bénin (Yorou et De Kesel, 2002), les communautés Bakweri du Mont Cameroun (Egbe et al., 2013) et une dizaine de communautés de la forêt montagneuse de Kilum-ljum, au nord-ouest du Cameroun (Teke et al., 2018).

Les CSC sont plus riches que les meilleurs légumes en vitamines, parmi lesquelles figurent toutes celles du complexe B (acide folique, ergostérine ou provitamine D, thiamine, riboflavine), mais aussi en minéraux (potassium, phosphore, cuivre, sodium, fer, manganèse, calcium), en fibres digestives (mannose, polysaccharides, cellulose, fibres chitineuses) et en protéines (Adebayo-Tayo et al., 2010 ; Johnsy et al., 2011 ; Kouame et al., 2018). Pour les populations locales, les CSC sont habituellement considérés comme des substituts aux protéines animales et connus comme "viande du pauvre ». Certes, les taux en protéines des CSC sont semblables à ceux des viandes; cependant, les protéines fongiques sont peu digestes, ne faisant pas des champignons de vrais substituts à la viande (Kalač, 2013 ; Feeney et al., 2014 ; Lister, 2015). L'usage et la consommation des champignons sauvages dépendent autant de leur appréciation culturelle.

Les champignons comestibles sont économiquement importants. La culture des champignons comestibles en Amérique du Nord, Europe et Asie est une source importante de revenus et alimente une chaîne de valeur très lucrative, créatrice de nombreuses petites entreprises et d'emplois (Arora et al., 2008). Dans les mêmes pays, les CSC sont pareillement collectés dans les forêts en automne par des mycologues amateurs et professionnels pour les vendre dans les supermarchés et restaurants. Cette chaîne de valeur constitue une vieille industrie multi-millionnaire en dollars américains (Oei, 1996 ; Arora et al., 2008). Au Burundi, les femmes réclament des droits sur les portions de terre où les Termitomyces présentent des boutons fongiques et les commercialisent le long des routes (Buyck, 1994). De plus, un groupe de CSC symbiotiques avec certaines essences endémiques des forêts humides (Gilbertiodendron, Microberlinia, Tetraberlinia) pourrait avoir de potentiels marchés internationaux. À certaines périodes de l'année, des CSC symbiotiques se développent abondamment sur des racines. Au regard de cette importance commerciale, toutes les parties prenantes en foresterie tropicale devraient être sensibilisées sur les relations symbiotiques entre les arbres et les CSC ectomycorhiziens (ECM). En revanche, la mise en marché des CSC symbiotiques tropicaux s'avère difficile.

Environ 400 espèces de champignons comestibles sauvages sont utilisées dans la pharmacopée traditionnelle, en Asie et en Afrique, pour soulager certaines affections telles que la pression sanguine, les tumeurs et les maladies virales (Oei, 1996 ; Boa, 2006 ; Tonjock et al., 2013). L'espèce comestible Tremella fuciformis Berkeley (biyae en bulu) et le tricholome Tricholoma matsutake (Vittad.) Sacc. (matsu-take en chinois) peuvent soigner la leucémie chez $65 \%$ des patients ; les polysaccharides de l'espèce Lentinula edodes (Berk.) Pegler, appelée shii-take, constituent un stimulant immunologique pour traiter l'hépatite virale et protéger le foie ; les espèces Ganoderma lucidum (Curtis ex Fr.) P. Karst. et $T$. fuciformis ont des effets antidiabétiques et antioxydants avérés (Oei, 1996 ; FAO, 2004).

Les premières identifications de champignons sauvages comestibles au Cameroun remontent au XIXe siècle (Hennings, 1895) et au début du XXe siècle (Heim, 1942). Quatre espèces de CSC ont alors été identifiées à Ebolowa, au Sud-Cameroun : Lepiota discipes, Marasmius (Fr.) spp., Mycena (Pers.) Roussel spp. et Lactarius gymnocarpus R. Heim ex R. Heim. Depuis lors, sans véritable projet national sur les macrochampignons sauvages comestibles, des inventaires et excursions mycologiques, épars dans l'espace et dans le temps, ont été menés un peu avant l'indépendance, mais surtout vers la fin du XXe siècle, et se sont poursuivis en ce début de $X X I^{e}$ siècle. L'objectif de cette étude était de réaliser une analyse de la diversité et de l'écologie des CSC dans le Grand Sud-Cameroun, ainsi que de leurs usages communautaires. 


\section{Méthodes}

\section{Sites d'étude}

La zone du Grand Sud-Cameroun est une vaste étendue forestière avec des îlots de savane, située en deçà du septième parallèle Sud et recouvrant les sept régions administratives : Centre, Est, Littoral, Nord-Ouest, Ouest, Sud et Sud-Ouest (figure 1). Les excursions mycologiques et les inventaires ont été conduits dans des portions de forêt humide, incluant le Mont Cameroun, les forêts de Bipindi-Lolodorf-Akom II, du Nord-Ouest et du Sud-Ouest, de même que la zone de transition Forêt-savane, le long de la route Obala-Ebebda en allant vers les savanes du Mbam et les Hauts-Plateaux de l'Ouest, jusqu'à Fundong et au village d'Abuh vers le Nigeria (figure 1 ).

Le paysage varie considérablement des hautes montagnes aux basses terres côtières en passant par des mosaïques de forêts secondaires et de savanes herbeuses

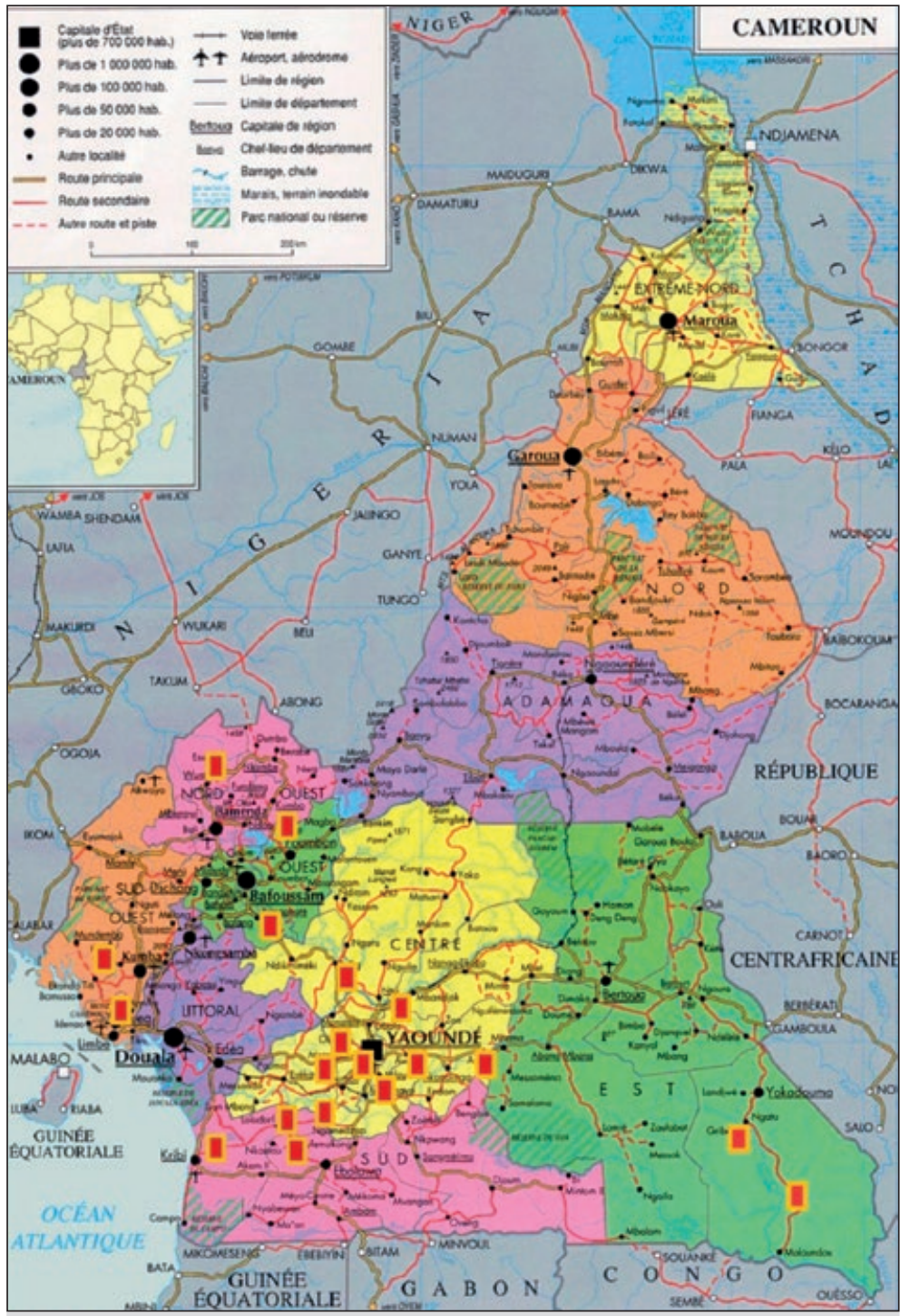

Figure 1.

Carte de localisation des sites d'étude dans le Grand SudCameroun (localisation approximative des sites sur la carte par des carrés rouges orangés). d'altitude moyenne. La végétation originelle est constituée de forêts denses sempervirentes, actuellement dégradées par des activités anthropiques telles que la culture itinérante sur brûlis et la coupe sélective d'essences forestières. Le climat est chaud et humide, avec des pluviométries abondantes mais variables selon la localité. L'humidité relative est généralement supérieure à 80 \%, toute l'année (Olivry, 1986). Au plan géologique, la zone varie des roches métamorphiques du Précambrien aux intrusions volcaniques récentes (Franqueville, 1973). Les caractéristiques physicochimiques des sols changent considérablement avec l'élévation, des cendres volcaniques riches en $\mathrm{N}$ et $\mathrm{P}$ aux sols ferralitiques, pauvres en éléments nutritifs.

\section{Groupes ethniques}

Une trame d'enquête adaptée a été conçue pour consulter les populations locales rencontrées pendant les excursions mycologiques (De Kesel et al., 2002 ; Härkönen et al., 2003). La trame d'enquête a été appliquée à tout type de personne afin d'évaluer les connaissances locales et le savoir-faire endogène sur les espèces locales de champignons utiles (variétés consommées et médicinales, saisonnalité, modes de préparation et de conservation, palatabilité, habitats, niches...). Après introduction et avec l'aide d'interprètes locaux, plusieurs ethnies locales ont été interviewées, notamment deux groupes de peuples autochtones (pygmées) Baka dans l'Est et Bagyeli dans le Sud, et de nombreux groupes ethniques Bantu incluant les peuples Bafia, Baka, Bakweri, Bamoun, Bassa, Bulu, Fang, Eton, Ewondo, Kom, Konabembe, Manguissa, Mvele, Ngumba, Sanaga, Yambassa et Yemba. Alors que les peuples Bantu interviewés vivent d'abord de l'agriculture itinérante sur brûlis, les pygmées pratiquent la collecte de produits forestiers autres que le bois, tels que les CSC, comme activité de subsistance principale.

\section{Enquêtes ethnomycologiques et identification des champignons}

Depuis 1996, des enquêtes ethnomycologiques ont été entreprises et se sont poursuivies jusqu'en 2015 dans de nombreux villages du Grand Sud-Cameroun (figure 1). D’après un catalogue de photographies constituées à partir d'études menées dans la région de Bipindi-Lolordof-Akom II, les premières espèces de CSC identifiées ont été montrées aux populations locales pour les reconnaître ou non. Elles confirmaient ensuite les espèces utiles et nous aidaient à les collecter au voisinage de leur terroir, à l'état frais. Les spécimens collectés localement ont été décrits à l'état frais, le même jour, puis séchés ou conservés dans une solution d'alcool éthylique diluée à $50 \%$, avant une description microscopique assurée au laboratoire. Parfois, lors d'abondantes collectes en saison pluvieuse, les spécimens ont été séchés dans un séchoir en bois à gaz méthane, pendant deux à trois jours, à environ $40{ }^{\circ} \mathrm{C}$. Sur le terrain, la consistance, l'odorat, et le goût ont représenté les principaux caractères observés.

Après photographie des échantillons, les caractères macroscopiques suivants ont été systématiquement décrits en après-midi, avant que le soleil décline : 
- couleur, forme, taille et aspect du chapeau, des lames et lamellules ou des tubes et du pied;

- ornementation et consistance du chapeau et du pied (présence d'écailles, de cônes, d'un anneau, de fibrilles) ;

- éventuels changements de couleur de la chair exposée à l'air ;

- mode d'insertion des lames par rapport au pied ;

- consistance des lamelles (ferme ou molle, veloutée ou visqueuse) ; caractérisation de la base (en sac ou en bourrelet, poilue ou glabre, avec ou sans débris organiques agglomérés) ;

- consistance, couleur, odeur et saveur de la chair ;

- aspect des spores observées avec une loupe de terrain (x 20) ; - mode de croissance (solitaire, en groupe, en touffe, en troupe, formant des ronds de sorcière) ;

- écologie (arbres à proximité, biotope, développement sur le bois, dans l'herbe, sur le sol ou sur un support particulier).

Au laboratoire, les principaux caractères examinés ont été : - la couleur de la sporée ;

- la forme et la taille des hyphes du chapeau, de la chair du pied ;

- la forme, la taille et les dimensions des spores ;

- la présence ou non d'éléments particuliers tels que les cystides (forme, taille) dans toutes les parties du champignon.

Ensuite, à l'aide de la documentation (Lãessoě et Del Conte, 2001) et de sites web ${ }^{1}$, les espèces ont été déterminées en comparant les descriptions fournies aux nôtres. Une partie des spécimens fongiques collectée est aujourd'hui conservée à l'Herbier national de Leiden, aux Pays-Bas. Des duplicatas ont été conservés aux laboratoires de microbiologie de l'IRAD, à Kribi et à Nkolbisson.

\section{Résultats}

\section{Habitats et niches des champignons sauvages comestibles}

En forêt humide du Grand Sud-Cameroun, les champignons sauvages comestibles sont collectés dans divers habitats, notamment, les jardins de case, les jeunes jachères à Chromoleana odorata ou Imperata cylindrica, les champs cultivés, les cacaoyères, les forêts secondaires, la forêt primaire, les peuplements forestiers formés de Cesalpiniaceae et Phyllantaceae, et les forêts-galeries à Uapaca spp. ou Gilbertiodendron dewevrei. Trois niches spécifiques ont été également observées, représentées par les termitières souterraines et aériennes pour les espèces de Termitomyces (R. Heim), le stipe de palmier à huile en décomposition pour Volvariella volvacea (Bul.) Singer, et un feutrage blanc de mycéliums pour Armillaria camerunensis (Henn.) Courtec. En général, les CSC ont été collectés sur la terre ferme.

\section{Biogéographie des champignons sauvages comestibles}

Les champignons sauvages comestibles se recrutent dans trois groupes trophiques et présentent une distribution biogéographique originale au plan national. En effet, les espèces saprotrophes et les Termitomyces sont rencontrés

1 https://www.efta-online.org/; www.indexfungorum. org ; www.mycodb.fr ; www.fungipedia.org dans tous les écosystèmes investigués. Mais les espèces symbiotiques, avec certaines essences forestières, se manifestent dans trois régions uniquement, Sud, Sud-Est et SudOuest, où elles forment des symbioses ectomycorhiziennes avec les racines d'espèces forestières appartenant à 13 genres: Afzelia, Anthonota, Berlinia, Brachystegia, Didelotia, Gilbertiodendron, Julbernardia, Microberlinia, Monopethalantus, Paraberlinia, Tetraberlinia, Touabouate et Uapaca. Ces essences forestières forment généralement des peuplements équiennes de petite, moyenne et grande taille, pouvant atteindre de longues distances, comme à Korup dans le Sud-Ouest pour les espèces de Microberlinia ou sur la route Lomié-Ngoïla dans le Sud-Est pour les espèces de Gilbertiodendron. Cependant, trois espèces ectomycorhiziennes des genres Afziela, Anthonotha et Berlinia n'apparaissent pas en peuplements ECM. La quatrième région est celle du Centre où l'on peut récolter des CSC ectomycorhiziens dans les forêts-galeries, le long de certains cours d'eau.

\section{Abondance et diversité des champignons sauvages comestibles}

Quatre-vingt-quatorze taxons de champignons sauvages comestibles ont été identifiés, parmi 32 familles et 41 genres, avec environ $17 \%$ de champignons (Termitomyces) mutuellement associés aux termites de la famille Macrotermitidae, 21,3\% d'espèces ectomycorhiziennes, et $61,7 \%$ d'espèces saprotrophes (tableau I).

Les Termitomyces de la famille des Lyophyllaceae sont les CSC les plus collectés et consommés (figure 2 ; photos 2-9 à 12). De nombreuses et diverses espèces de saprotrophes sont également consommées (photos 2-1 à 8). Les espèces de CSC les plus appréciées incluent $A$. camerunensis (Henn.) Courtec. (photo 2-1), V. volvacea (Bul.) Singer (photo 2-4), Lactifluus gymnocarpus Verbeken (photo 2-15), toutes les espèces de Termitomyces et Cantharellus (photos 9 à 16 ; photos 2-13 et 16). Certaines espèces de CSC les moins collectées sont celles des genres Collybia (Fr.) Staude, Gymnopilus (P. Karst), Pluteus, Psathyrella, Polyporus (Adans.), Ramaria (Fr ex Bonord), Russula, Schizophyllium et Trichaleurina. Les CSC ectomycorhiziens sont peu collectés pour l'alimentation fongique locale. Par exemple, les espèces du genre Amanita et les bolets sont rarement collectés pour la consommation par les populations locales (figure 2).

Les Termitomyces sont les seules espèces de CSC vendues, fraîches ou séchées, le long des routes ou dans les marchés conventionnels. Aucune espèce de CSC n'est actuellement exportée ni commercialisée dans les supermarchés, hôtels et restaurants camerounais.

Quinze espèces de champignons sauvages comestibles sont aussi collectées pour leurs propriétés médicinales, incluant les genres suivants : Agaricus (L.), Auricularia, Chlorophyllum, Cookeina (Kuntze), Cyathus, Daldinia, Flavolus, Ganoderma, Pleurotus (Fr.) P. Kumm., Tremella, Termitomyces et Russula. Une seule espèce, Chlorophyllum molybdites Meyer (Fr.) Mass., a été reconnue par certaines communautés Bakweri de Buéa (Sud-Ouest-Cameroun), comme provoquant des perturbations et des hallucinations. C. molybdites y est utilisée pour des incantations mystiques. 
Tableau I.

Liste des espèces de champignons sauvages comestibles en forêt humide du Grand Sud-Cameroun par groupe trophique, site de collecte et habitat.

\section{Noms scientifiques}

Agaricus sp1

A. campestris $\mathrm{L}$.

Armillaria camerunensis

(Henn.) Courtec.

Auricularia auricula Hooker

Auricularia cornea (Beeli)

A. polytricha (Mont.) Chlorophyllum molybdites **

(Meyer : Fr.) Mass.

Collybia cf. allegretti

Collybia sp1

C. aurea (Beeli)

C. occidentalis

Cookeina sulpices* (Berk.)

Kuntze

C. tricholoma (Mont.) Kuntze

C. globosa* (Douanla-Meli)

C. brunneofibrillosus*

C. comatus* (O.F. Müll.) Pers.

Cyathus striatus*

(Huds. ex Pers.) Willd.

Dacryopinax spathularia*

(Schweinitz) G.W. Martin

Daldinia* eschscholtzii

(Ehrenb.)

Entoloma camerunensis

Entoloma sp1

Flammulina velutipes (Curtis)

Singer

Favolus brasiliensis

Ganoderma applanatum *

(Pers.) Pat.

G. lucidum* (Curtis) P. Karst.

Grifola frondosa (Dickson) Gray

Gymnocarpus aureobruneus

Laetiporus baudonii* (Pat.)

Ryvarden

Lentinus citrinopileatus

L. polychrous

L. tigrinus (Fr.) Fr.

Lepiota sp1

Lepiota discipes

Lepiota cristata (Bolton)

P. Kumm.

Macrolepiota procera (Scopoli)

Singer

\section{Lieux de collecte}

Abuh, Fundong,

Kom village,

Nkolbisson

Ebom, Megong,

Ebolowa

Fundong, Batibo

Bityili, Akonolinga

Nyangong

Flancs du Mont

Cameroun, Nkolbisson

Ebom

Gribé, Nyangong,

Tolong

Ebom, Nyangong,

Akonolinga,

Mbalmayo

Akonolinga,

Mbalmayo

Ebom, Mbalmayo

Bityili, Ebolowa,

Fundong

Okola, Lobo, Nlong,

Mefomo

Bityili

Nkol-Foulou, Kumba

Ebom

Kumba,

Batibo, Fundong

Nyangong

Nkolbisson

Abuh, Batibo,

Mbalmayo

Kom, Kumba, Mefomo

Ebom, Nkolbisson

Buea

Buea

Bafia

Nkolbisson, Ntui

Ebom

Ebolowa, Nyangong

Bityili, Ebolowa Si

Nkolbisson

\section{Nom local et tribu}

Cocobianco (Pidgin

english)

Kôndô (Bulu)

Cocobianco (Pidgin)

Biyae (Bulu, Ewondo)

Mvufulu alen (Bulu)

Abon Melen

Anamedu

(Kounabembe),

Tôlong (Bulu, Ewondo,

Bagyeli)

Oyoyonguogue (Bulu)

Onon vio (Ewondo)

Biyaé (Bulu)

Assakokutou (Bulu)

Vetuiti ou Cocobianco

(Pidgin)

Ongô

Kep (Kom),

Cocobianco (Pidgin)

Cocobianco (Pidgin)

Etekum (Bulu)

Ebwebwe (Bawkeri)

Yellow cocobianco

Bekoupassubu (Bulu)

(B)

Vio bikorok (Ewondo)

\section{Sur souches ou troncs d'arbres en décomposition}

Sur sols sous forêt secondaire et cacaoyères

\section{Commentaires sur l'habitat}

Sur sols nus sous pieds d'Eucalyptus sp. ou dans les jachères ou gazons

Sur souches d'arbres en décomposition

En ronds de sorcière sur sols volcaniques ou fumier de poule

En touffes sur souches de bois mort

Sur souches ou bois mort dans tous les écosystèmes forestiers

En touffes sur bois mort en forêt secondaire et dans la Réserve forestière de Mbalmayo

À l'état dispersé ou en groupe sur bois morts ou débris végétaux en forêt non dégradée et sur troncs d'arbres abattus dans les champs d'arachides

\section{Sur sols forestiers rouges}

Sur sols humides ou litière humide de débris végétaux

Sur troncs d'arbres en décomposition

Sur souches de bois mort

Sur souches de bois sec

Sur souches de bois sec ou en décomposition

Sur bois vivant

Sur souches de bois mort

En touffes sur souches d'arbres morts

Solitaire ou en petites troupes sur sols engazonnés 
Tableau I (suite). Noms scientifiques

\begin{tabular}{|c|c|}
\hline $\begin{array}{l}\text { Marasmiellus candidus (Fries) } \\
\text { Singer }\end{array}$ & Ebom \\
\hline Marasmius katangensis Singer & $\begin{array}{l}\text { Nyangong, Mengong, } \\
\text { Zoaetele }\end{array}$ \\
\hline Phallus indusiatus* & Nkol-Foulou, Okola \\
\hline Psathyrella arrombonatus & Okola, Mefomo, Nlong \\
\hline P. candolleana (Fr.) Maire & Obala \\
\hline P. cf. ovaticystis & Obala \\
\hline $\begin{array}{l}\text { Pleurotus flabellatus (Fr.) } \\
\text { P. Kumm. }\end{array}$ & Nyangong, Ebom \\
\hline $\begin{array}{l}\text { P. ostreatus (Jacq. ex Fr.) } \\
\text { P. Kumm. }\end{array}$ & $\begin{array}{l}\text { Balikumbat, Batibo, } \\
\text { Kumba, Gribé, } \\
\text { Nkolbisson }\end{array}$ \\
\hline \multicolumn{2}{|l|}{ P. pulmonarius (Fr.) Quél. } \\
\hline P. tuber-regium* (Fr.) Singer & Partout \\
\hline P. sajor-caju (Fr.) Fr. & $\begin{array}{l}\text { Batibo, Buea, Ebom, } \\
\text { Gribé, Nkolbisson }\end{array}$ \\
\hline P. squarrosulus Mont. & $\begin{array}{l}\text { Kribi, Nkong-abok, } \\
\text { Okola }\end{array}$ \\
\hline Pluteus cf. griseoroseus & Ebom, Lobo, Okola \\
\hline \multicolumn{2}{|l|}{ Schizophyllum commune Fries } \\
\hline $\begin{array}{l}\text { Stereum hirsutum* (Willd. : Fr.) } \\
\text { Gray }\end{array}$ & \multirow[t]{3}{*}{$\begin{array}{l}\text { Kribi, Nkolbisson, } \\
\text { Lobo, Ngomedzap }\end{array}$} \\
\hline Trametes versicolor* (L.) Lloyd & \\
\hline $\begin{array}{l}\text { Tremella fuciformis* Berk. } \\
\text { T. mesenterica Retz. }\end{array}$ & \\
\hline $\begin{array}{l}\text { Trichaleurina javanica (Rehm) } \\
\text { M. Carbone }\end{array}$ & Ebom, Mefomo, \\
\hline $\begin{array}{l}\text { Volvariella caesiotincta } \\
\text { P.D. Orton }\end{array}$ & Abuh, Fundong, Kom \\
\hline V. gloiocephala (DC.) Wasser & \\
\hline V. volvacea (Bul.) Singer & $\begin{array}{l}\text { Ebom, Ngomedzap, } \\
\text { Okola, Obala }\end{array}$ \\
\hline
\end{tabular}

\section{Nom local et tribu}

Mvufulu alen (Bulu)

Abon si (Bulu)

Sesa Mbel (Eton),

Mbel (Eton),

Oyoyonguogue (Bulu), Mbel (Eton), Adikuju (Bagyeli)

Mvufulu (Bulu)

Bikoko biyogo (Ewondo), Cocobianco (Pidgin)

\section{Uboôh (Kom),} Cocobianco (Pidgin English)

\section{Dunda (Bulu) ou Duna}

(Eton) ou Dundé (Bayeli),

Essas (Kounabembe),

Koutou (Baka)

Epour (Kounabembe),

Esusuk (Bulu),

Agwuog (Kom)

Bikoko biyogo (Ewondo, Bassa, Batanga, Eton) Zibminkôk (Bulu)

Ebom Mefomo

Biyae (Bulu, Bagyeli, Ewondo, Eton)

Nyeng (Bulu, Ewondo),

Ugwuog (Kom),

Cocobianco (Pidgin)

Etôk melen (Bulu,

Ewondo) Ingouga (Eton)

\section{Commentaires sur l'habitat}

Sur gazon

Sur un feutrage mycélien abondant en forêt secondaire

Sur sols nus autour de souches et troncs d'arbres abattus En touffes sur de vieilles souches de palmier à huile ou autres arbres en décomposition

Sur manguier et souches de bois mort

En grosses touffes sur le tronc et branches de manguier et souches de bois en décomposition

Sur souches en décomposition

Sur tubercule sclérotique

Sur souches de bois mort

Sur troncs, vieilles souches et branches tombées à terre En touffes sur souches de bois en décomposition Sur souches de bois mort ou branches de safoutier) (Dacryodes edulis

Sur bois en décomposition en forêt secondaire ou sur souches de bois mort

En petites troupes sur sol humide

Solitaire sur sol

Sur troncs de palmier à huile (Elaeais guineensis)

en décomposition

Champignons sauvages comestibles mutuellement associés aux termites (Termitomyces)

Termitomyces sp1

Termitomyces sp2

Ebom

Babe (Bulu)

Topkwé (Bulu)

Solitaire au sol

Termitomyces aurantiacus

Ntui, Obala

Mbel (Eton),

(R. Heim) R. Heim

Nlom (Bulu, Ewondo)

T. clypeatus (R. Heim)

T. entomoloides var Gribé

Termitomyces fuliginosus

(R. Heim)

Termitomyces globulus

\section{Gribé}

(Heim \& Goss-Font.)

Obala, Batchenga Mfumu (Bulu)

Sur sols ferralitiques rouges forestiers

Sur sols sablonneux de savane à Imperata cylindrica, sur sols forestiers ou de jachère, ou à l'état éparpillé dans jeunes champs d'arachides

T. le-testui (R. Heim)

Obala, Ntui 
Tableau I (suite). Noms scientifiques

\section{T. mammiformis (R. Heim)}

T. mboudaeina Mossebo

T. microcapus (Berk. \& Broome)

R. Heim

T. medius

\section{T. robustus (Beeli) R. Heim}

T. schimperi (Pat.) R. Heim

T. striatus f. grisumboides

T. titanicus (Pegler \& Piearce)

Amanita vaginata (Bull.) Lam.

A. rubescens (Pers. ex Fr.) Gray

Cantharellus tenuis (Heinem.)

(Otoye)

C. miniatescens (Heinem.)

C. congolensis (Beeli)

C. foridulus (Heinem.)

C. isabellinus (Adans. ex Fr.)

C. platyphyllus (Heinem.)

C. pseudocibarius (Henn.)

C. rufopunctatus (Beeli.)

Heinem.

Craterellus cornucopioides

(J. Schröt. in Cohn)

Lactafluus gymnocarpus

(Verbeken) Verbeken

L. rubroviolascens (R. Heim)

Gomphus sp1

Ramaria sp1

Russula cellulata (Buyck)

R. congoana (Buyck)

Suillus granulatus (L.) Roussel)

Strobilomyces strobilaceus

(Scop.) Berk. (1851)

\section{Lieux de collecte}

Balikumbat, Fundong

Koutaba, Mbouda)

Akonolinga Ebom

Gribé Koutaba Mbouda

Nkolbisson, Nlong

Gribé

Balikumbat Ebom

Gribé

Batchenga Minkama

Monatele, Obala

Abuh, Bafoussam

Bamengoum, Kom,

Mbouda
Puo (Ngemba

Cocobianco (Pidgin)

Puo (Ngemba)

Osé (Bulu, Ewondo,

Eton,Mvele) ou Sewo

(Bagyeli) Tassok

(Kounabembe)

Mebolmekoul

(Kounabembe)

Touloubemba (Baka)

Avam si (Bulu)

Puo wetti (Kom)

Mbel mesil (Eton,

Manguissa)

Puo (Ngemba)

Puo (Ngemba)

Lemkwalih (Kom)

\section{Commentaires sur l'habitat}

Solitaire sur sols ferralitiques rouges

Sur sols ferralitiques rouges de forêt ou de cacaoyère ou sur gazon

\section{Sur sols sablonneux de savane}

Sur sols ferralitiques rouges de montagne

Sur sols sablonneux de savane ou sols ferralitiques rouges de jachère à Chromoleana odorata

Dans de petits peuplements d'Uapaca sp. au bord de la rivière Nyong

Entre les pieds de petits peuplements mixtes d'ekop ${ }^{\psi}$ et autres arbres ectomycorhiziens
Nyavem ou

Otsetsa (Bulu) ou

Nyambembo (Bayeli)

Otsetsa mebeum

(Ewondo) ou

Mbel assam (Eton)

Bingobidong (Bulu)

Nkolbisson
Bingobidong (Bulu)

Anpembo(Bagyeli)

\section{Nyumelane (Bulu, Fang)}

\section{Bityili Ebimimbang}

Ebom, Nyangong

Gribé

Gribé, Mvie Bekono

Ezendouan

Nyangong, Ebom

Ebimimbang

Gribé

\section{Ebimimbang \\ Bityili Ebimimbang \\ Ebom, Nyangong \\ Nyangong \\ Ebom Nyangong \\ Okola, Lobo \\ Bityili Nyangong Ebom}

Bityili

Les espèces suivies d'un astérisque sont surtout utilisées dans la pharmacopée traditionnelle.

* Ekop est un nom générique octroyé par les prospecteurs au Cameroun et au Gabon aux espèces de genres des Cesalpiniaceae sans nom (Letouzey et Mouranche, 1952).

* Champignons sauvages comestibles surtout utilisés dans la pharmacopée traditionnelle.

** Champignon sauvage à comestibilité controversée, avec possibilité de toxicité, à dire d'acteurs.

Autour des racines-échasses en forêts-galeries

En troupes au pied d'Uapaca sp. près d'un cours d'eau

Sur sols ferralitiques rouges et sablonneux de peuplements forestiers

Autour des racines-échasses en forêts-galeries

Sur sols engazonnés d'une petite pinède devant la direction générale de l'IRAD

Dans des peuplements forestiers mixtes d'espèces

de Cesalpiniaceae et Uapaca sp. 


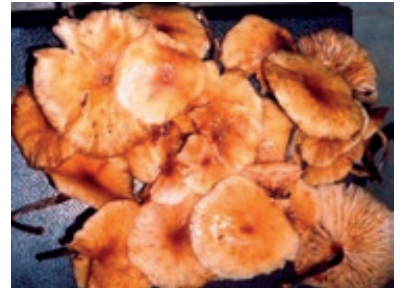

1) Armillaria camerunensis (Henn.) Courtec.

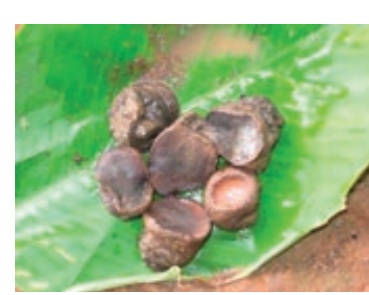

2) Trichaleurina javanica (Rehm) M.Carbone

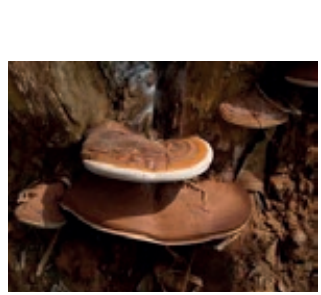

3) Ganoderma applanatum (Pers.) Pat.

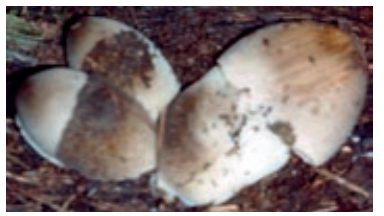

4) Volvariella volvacea (Bul.) Singer

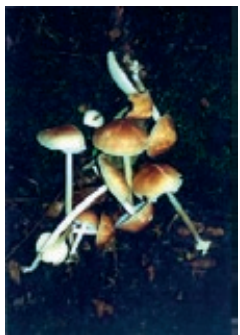

5) Marasmius katangenisis Singer

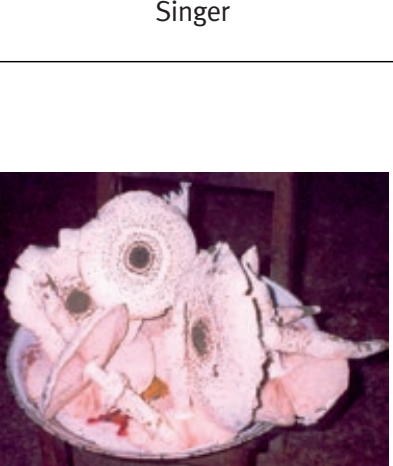

9) Termitomyces schimperi (Pat.) R. Heim

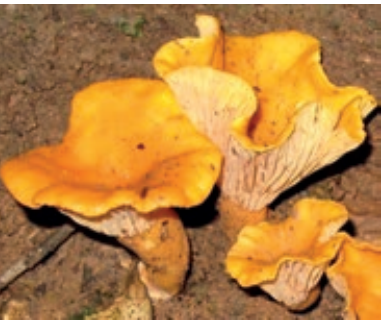

13) Cantharellus rufopunctatus (Beeli) Heinem.

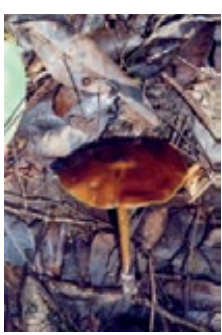

6) Entoloma camerounensis

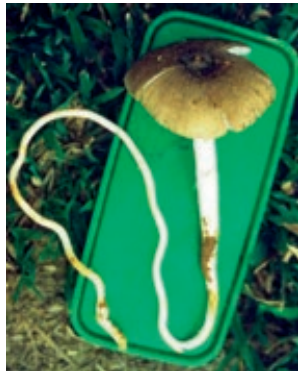

10) Termitomyces striatus (Beeli) Heim

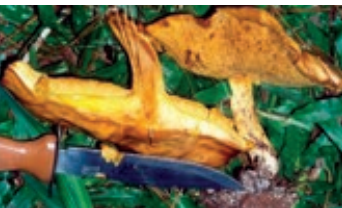

14) Suillus Granalutus (L.) Roussel

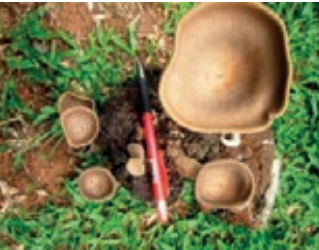

7) Pleurotus tuber-regium (Fr.) Singer

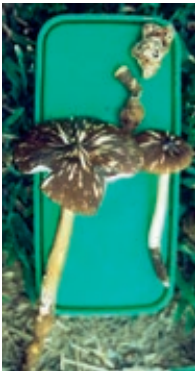

11) Termitomyces robustus (Beeli) R. Heim

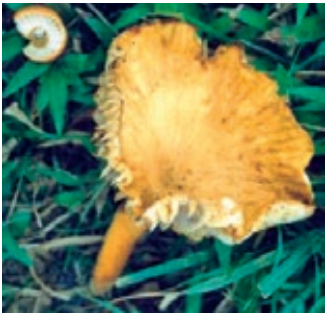

15) Lactifluus gymnocarpus (Verb.) Verbeken

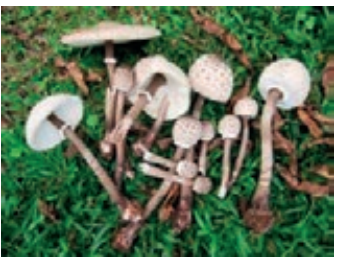

8) Macrolepiota procera (Scopoli) Singer

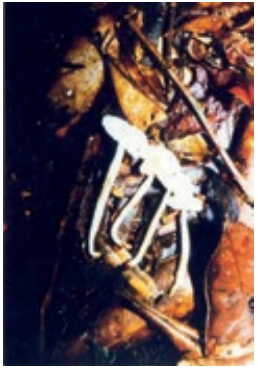

12) Termitomyces microcarpus (Berk. \& Br.) Heim

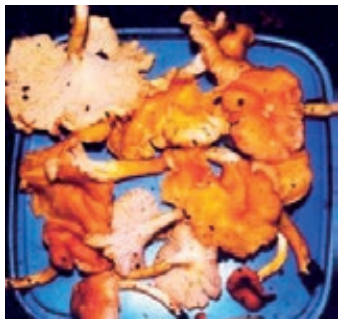

16) Cantharellus miniatescens Heinem.

Photos 2.

Compendium de quelques photographies de champignons sauvages comestibles du Grand Sud-Cameroun. Champignons sauvages comestibles saprotrophes, Termitomyces et ectomycorhiziens. Photos N. Onguene Awana. 


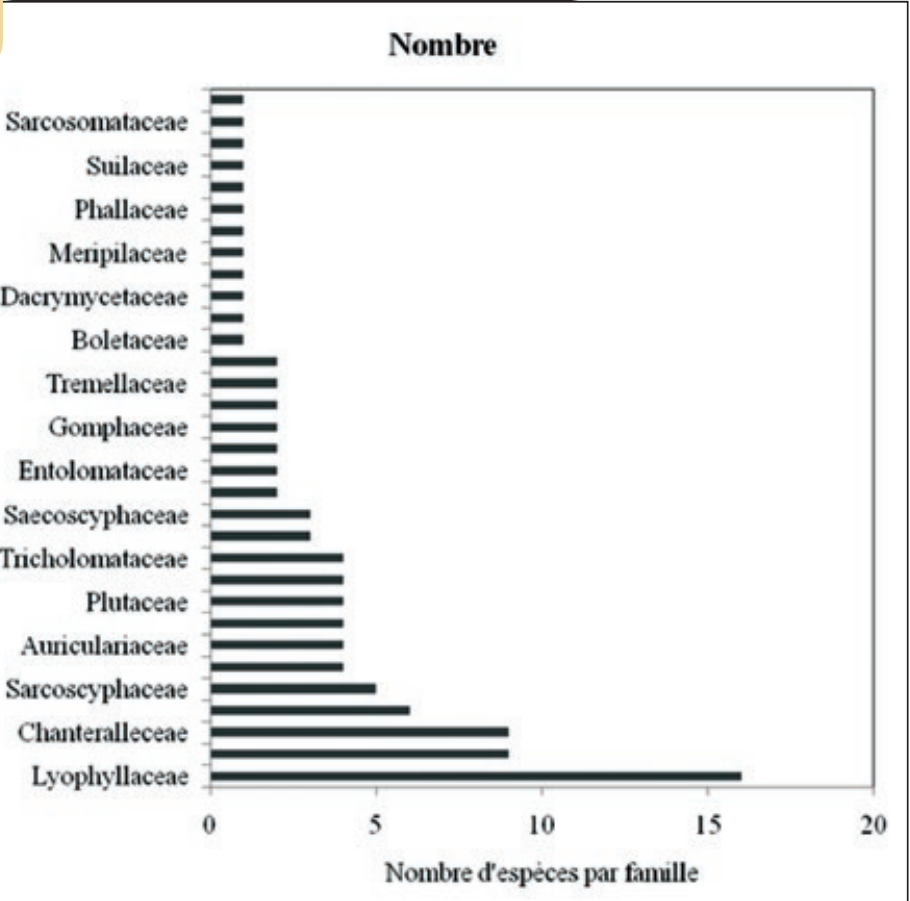

Figure 2.

Abondance relative des espèces de champignons sauvages comestibles des forêts humides du Grand Sud-Cameroun par famille.

\section{Discussion}

Quatre-vingt-quatorze espèces de champignons comestibles sauvages, appartenant à trois groupes trophiques, ont été identifiées et répertoriées en forêt humide du Grand Sud-Cameroun (tableau I ; figure 1). Cette forte diversité spécifique est disproportionnée au plan trophique, avec environ $61,7 \%$ de saprotrophes, $21,3 \%$ d'espèces ectomycorhiziennes et $17 \%$ de Termitomyces. Les espèces comestibles de la famille des Lyophyllaceae (Termitomyces) sont les plus collectées et consommées (figure 2). Les 20 espèces ectomycorhiziennes de CSC appartiennent à seulement cinq genres - Cantharellus, Lactarius, Lactifluus, Ramaria et Russula - et sont symbiotiquement associées à deux familles botaniques seulement, Cesalpiniaceae et Phyllantaceae, pour former des ectomycorhizes (Onguene et Kuyper, 2001 ; Boa, 2006 ; Ebenye et al., 2016). Nos résultats sont semblables à ceux obtenus au Gabon (62 espèces), au Nigeria (78 espèces) et en RD Congo (150 espèces) (Boa, 2006 ; Eyi et al., 2011 ; Ba et al., 2014). Ceci dénote une forte homogénéité fongique au sein d'un continuum floristique forestier très diversifié d'Afrique de l'Ouest vers l'Afrique centrale. Cependant, cet inventaire incomplet n'inclut pas les espèces de CSC des régions de l'Adamaoua et du Grand Nord (figure 1). Par ailleurs, ces résultats spatio-temporels ne proviennent que d'une vingtaine de localités. La documentation de la biodiversité des CSC du Cameroun ou d'Afrique centrale reste donc largement incomplète, en dépit de leur quadruple importance alimentaire, nutritionnelle, monétaire et écologique. Les communautés «Awing » au Nord-Ouest-Cameroun valorisent plus d'une soixantaine d'espèces de champignons comestibles dont trois sont utilisées comme aliment et médicament : Termitomyces titanicus Pegler \& Piearce, Laetiporus baudonii (Bull. : Fr.) Murrill et Ganoderma sp. (Tonjock et al., 2017). Les groupes socio-linguistiques des MontsKouffè, Peuls, Bètamaribè et Lopka, au Bénin, consomment une dizaine de saprotrophes (Fadeyi et al., 2017). Ce mode de consommation des CSC a été aussi observé en Éthiopie (Dejene et al., 2017). À l'opposé, les peuples Bassar et Kabyè, riverains du Parc national Fazao-Malfakassa (PNFM) au Togo (Afrique de l'Ouest), consomment de nombreuses espèces de champignons ectomycorhiziens dont Amanita loosii Beeli, Russula oleifera Buyck et Cantharellus congolensis Beeli (Kamou et al., 2017).

À l'exception des chanterelles et de Pleurotus ostreatus (Jacq.) P. Kumm., la cuisine mycologique camerounaise semble totalement différente de celle des pays européens. Dans la cuisine européenne, ce sont surtout des espèces des genres Agaricus, Agrocybe (Fayod), Amanita, Boletus (L.), Calocybe, Coprinus (Kühner ex Donk), Cantharellus, Craterellus qui sont collectées et consommées (FAO, 2004). Les espèces de ces genres sont considérées comme les champignons les plus appréciés par les Européens. Cependant, dans ces genres comme Agaricus et Boletus (au sens large), Coprinus et surtout Amanita, il existe des sosies toxiques, dont certains mortels. Au Cameroun, les meilleurs choix sont toutes les espèces de Termitomyces, l'espèce saprotrophe $A$. camerunensis et le champignon de paille $V$. volvacea, ainsi que la dizaine d'espèces de chanterelles. L'appréciation culinaire des CSC dépend fortement des traditions et cultures. Au Cameroun, les pygmées Baka de l'Est consomment plus de saprotrophes que les Bagyeli du Sud-Cameroun dont la cuisine mycologique est surtout composée de chanterelles et lactaires.

L'intérêt majeur des champignons réside dans leur saveur inégalable. C'est la raison essentielle de leur succès auprès des gourmets et des gastronomes. En forêt humide du Grand Sud-Cameroun, toutes les espèces de Cantharellus (chanterelles ou girolles), les Termitomyces, le champignon de paille $V$. volvacea et l'espèce $A$. camerunensis sont les CSC les plus appréciés des populations locales. Plusieurs espèces des genres Auricularia, Cookeina, Lactarius, Lentinus, Marasmius, Pleurotus et Tremella sont couramment collectées et directement consommées par les populations locales. D'autres espèces peu consommées incluent les genres Collybia, Gymnopilus, Pluteus, Ramaria, Russula, Psathyrella, Polyporus, Schyzopyllium et Trichaleurina. En général, les espèces des genres Amanita et les bolets sont rarement collectés pour la consommation. Les populations autochtones (pygmées) estiment que le changement de couleur des bolets après leur brunissement ou exposition à l'air est le signe évident de leur forte toxicité. Cette observation a été aussi faite en Tanzanie (Härkönen et al., 2003). Pourtant, certaines espèces d'amanites telles que l'amanite de César (oronge) sont d'excellentes espèces comestibles depuis l'époque romaine ainsi que le bolet de Bordeaux (Boletus edulis Bull.). Le plus grand nombre d'espèces de CSC au monde est constitué d'espèces de CSC symbiotiques, qui comptent environ 10000 espèces, avec une incidence commerciale élevée (Fortin et al., 2008). 
Parallèlement à la forte diversité d'espèces de champignons sauvages comestibles, leurs habitats et niches varient également fortement et spécifiquement : dans la nature, le champignon de paille pousse uniquement sur les troncs de palmier à huile en décomposition ; Pleurotus tuber-regium (Fr.) Singer ne se développe que sur un tubercule formé de mycéliums entrelacés et compacts, transportable à domicile, et qui, arrosé d'eau, donne naissance à des boutons fongiques puis des carpophores (photo 2-7) ; les chanterelles et lactaires n'apparaissent qu'en symbiose avec les racines de certaines essences forestières formant des peuplements forestiers équiennes dans les régions du Sud, Sud-Est et Sud-Ouest du Cameroun ou dans les forêts-galeries dans les régions du Centre, de l'Ouest et dans le Grand Nord (Newbery et al., 1988 ; Onguene, 2000 ; Onguene et Kuyper, 2001 ; Ebenye et al., 2016 ; Njouonkou, 2011 ; Njouonkou et al., 2016 ). À l'opposé, l'habitat et les niches des Termitomyces (termitières) varient avec leur taille et l'écosystème. Les Termitomyces de petite taille abondent en zone de forêt humide, les espèces de taille moyenne pullulent dans les savanes des Hauts-Plateaux de l'Ouest et, dans la zone de transition forêt-savane, la grande espèce dominante et commerciale est Termitomyces schimperi (Pat.) R. Heim. En somme, T. schimperi est panafricaine, apparaissant dans la quasi-totalité des pays au sud du Sahara (Boa, 2006). Ce sont surtout les espèces de Termitomyces qui constituent en majorité le marché local du CSC au Cameroun, à l'exception des espèces exotiques, rencontrées dans certaines grandes surfaces à Yaoundé et Douala (Djomene et al., 2017). En effet, les espèces de Termitomyces sont récoltées, séchées et vendues par les femmes. Seule T. schimperi est souvent vendue, à l'état frais, le long de la route Obala-Ebebda et dans certains marchés de Yaoundé, à 100-200 FCFA le spécimen (Onguene, obs. pers.). Toutes les autres espèces sont directement consommées à l'état frais après collecte en brousse.

La majorité des champignons sauvages comestibles sont des saprotrophes appartenant à une vingtaine de genres. Cette forte diversité de CSC est typique de la forêt humide de basse altitude ; 21,3\% de CSC symbiotiques ont été observés sur les racines d'essences forestières ectomycorhiziennes (Onguene et Kuyper 2001 ; Ba et al., 2014 ; Ebenye et al., 2016). Cependant, la grande majorité des taxons ECM identifiés ne sont généralement pas consommés localement, comme au Togo chez les communautés voisines du Parc national de Fazao-Malfakassa (Kamou et al., 2017). En particulier, les amanites et les bolets ne sont consommés ni par les Bantu ni par les pygmées, à l'exception d'Amanita rubescens par des communautés Mvele d'Akonolinga et de Russula congoana par les communautés Kounabembe de l'Est-Cameroun (tableau I).

La soixantaine de saprotrophes comestibles sont potentiellement cultivables. Cela représente une plus-value potentielle des saprotrophes par la valorisation de déchets agricoles du Cameroun (maïs, riz, coton, bagasse de canne à sucre, parches de café), avec des effets potentiels immédiats sur le commerce et l'amélioration des revenus agricoles des femmes rurales et des jeunes désœuvrés. Au Vietnam, le champignon de paille $V$. volvacea, sauvage au Cameroun, appelé localement etôk melen en bulu, ou ingouga en eton (tableau I ; photo 4), est intégré à la production du riz (Oei, 1996). Cependant, partout où les saprotrophes sont cultivés, il est nécessaire de disposer d'une provision stable de semences de champignons, appelées "blanc de semis". $\mathrm{Au}$ Cameroun, des centres de production du blanc de semis n'existent pas encore, ce qui peut menacer le capital sur pied. L'expansion du shii-take (Lentinula edodes) au Qingyan en Chine, pays phare du champignon, a entraîné l'épuisement des forêts locales ayant fourni le bois pour la culture de cette espèce comestible.

La vingtaine d'espèces symbiotiques de chanterelles et de lactaires pourrait-elle poser un problème de conservation des peuplements forestiers du Cameroun, en cas d'exploitation et d'exportation massive ? Cette interrogation mérite de s'y pencher. En effet, dans les forêts du Parc national de Korup, de la Réserve de biosphère du Dja et partout dans les forêts du Sud et du Sud-Est du Cameroun, de nombreux peuplements de taille moyenne et grande subsistent et sont exploités par les populations locales pour récolter des chanterelles et lactaires en saison pluvieuse. Elles ignorent pour le moment que ces espèces présentent une plus-value à l'exportation. En effet, un kilogramme de chanterelles coûte en moyenne 26,5 à 28,5 euros en France ou en Allemagne ${ }^{2}$. Les CSC symbiotiques d'Afrique peuvent donc aider à sauvegarder les forêts humides d'Afrique centrale. Cependant, il conviendrait de susciter une prise de conscience des parties prenantes quant à l'impact de la symbiose ectomycorhizienne sur la conservation forestière. En général, les espèces de champignons symbiotiques ne sont pas cultivables. Mais certaines espèces ECM telles que les truffes sont cultivables par inoculation de jeunes truffiers, par simple trempage (Hall et al., 1998). La régénération forestière avec des essences natives du Cameroun pourrait bénéficier d'une telle approche. Cependant, l'Agence nationale de régénération forestière (ANAFOR) ne dispose pas encore des outils d'inoculation ectomycorhizienne d'espèces des genres Afzelia, Gilbertiodendron ou Tetraberlinia.

Certaines espèces de champignons sauvages comestibles peuvent avoir des propriétés médicinales. Au cours de cette étude, 15 espèces de CSC ont été également rapportées comme ayant des vertus médicinales. En général, il s'agissait d'un caractère secondaire et connu seulement par un petit nombre de personnes interviewées. Les résultats ont parfois été cohérents avec les observations faites dans d'autres pays. Au Bénin, au Nigeria et à Madagascar, quelques espèces de CSC citées au Cameroun semblent prévenir ou même soigner plusieurs maladies telles que les inflammations, les rhumatismes, l'infertilité féminine et les cancers. Le sclérote de $P$. tuber-regium paraît avoir de nombreuses vertus. Au Nigeria, à Madagascar et au Sud-Cameroun, il est respectivement employé comme antipoison ou pour soigner le mal de poitrine et les ulcères d'estomac. Au Burundi, les meules de termitières servent à soigner la crise cardiaque (Buyck, 1994). C. molybdites (G. Meyer) Mass., signalé comme provoquant des hallucinations, y est utilisé pour des incantations mystiques. La palatabilité de cette

2 https://rnm.franceagrimer.fr/prix?CHAMPIGNONS 
espèce reste controversée. Elle est consommée au Ghana et au Burkina Faso mais pas au Congo et dans plusieurs autres pays d'Afrique occidentale et centrale (De Kesel et al., 2002 ; Boa, 2006 ; Guissou et al., 2015).

In fine, deux principaux services écosystémiques sont fournis par les champignons sauvages comestibles du Cameroun : le service d'approvisionnement alimentaire et médicinal et le service économique d'apport de revenus. Deux besoins urgents de recherche s'imposent afin de préserver cette importante partie de la biodiversité des sols, sous la forme notamment d'études de la biodiversité des macrochampignons sauvages comestibles et du développement de la culture du champignon de paille ( $V$. volvacea).

\section{Remerciements}

Les auteurs remercient le Gouvernement camerounais à travers l'Institut de recherche agricole pour le développement (IRAD). L'Organisation néerlandaise pour l'avancement de la science (NWO), dans son programme Biodiversité des écosystèmes perturbés, a financé les travaux sur les champignons sauvages comestibles du Sud-Cameroun. Dans différentes localités, de nombreuses femmes rurales, associées à leurs époux et enfants, ont accueilli avec enthousiasme et convivialité les chercheurs, les vulgarisateurs et les étudiants. Qu'ils en soient remerciés. Véronique Anaba et plusieurs assistants de terrain ont été les premiers ouvriers pour l'avènement de ce travail. Leur dévouement est aussi remercié. Deux évaluateurs anonymes ont formulé de précieuses remarques et suggestions sur une version du manuscrit. Qu'ils trouvent ici la gratitude des auteurs.

\section{Références bibliographiques}

Adebayo-Tayo B. C., Ekerete S. B., 2010. Effect of cultural conditions on exo-biopolymer production and biomass growth by Pleurotus sajor-caju (Fr.) Quel, a Nigerian edible mushroom. Advances in Food Sciences, 32 (2): 75-81.

Arora D., Susie M., Dunham S. M., 2008. A New Commercially Valuable Chanterelle Species, Cantharellus californicus sp. nov., Associated with Live Oak in California, USA. Economic Botany, 62 (3): 376-391.

Ba A. M., McGuire K. L., Diédhiou A. G., 2014. Ectomycorrhizal symbioses in tropical and neotropical forests. Boca Raton, USA, CRC Press.

Boa E., 2006. Produits forestiers non ligneux 17. Champignons comestibles sauvages. Vue d'ensemble sur leurs utilisations et leur importance pour les populations. Rome, Italie, Publications FAO, 170 p. https://www.efta-online.org/ termitomyces-schimperi/

Buyck B., 1994. Ubwoba : Les champignons comestibles de l'Ouest du Burundi. Bruxelles, Belgique, Administration générale de la coopération au développement, Publication agricole $\mathrm{n}^{\circ} 34$.

De Kesel A., Codjia J. T. C., Yorou N. S., 2002. Guide des champignons comestibles du Bénin. Bruxelles, Belgique, Jardin botanique national de Belgique, 272 p.
Dejene T., Oria-de-Rueda J. A., Martín-Pinto P., 2017. Wild mushrooms in Ethiopia: A review and synthesis for future perspective. Forest Systems, 26 (1).

Dijk H. van, Onguene N. A., Kuyper T. W., 2003. Knowledge and utilization of edible mushrooms by local populations of the rain forest of South Cameroon. Ambio, 32 (1): 15-23.

Djomene Y. S., Foudjet E. A., Fon D. E, Ninkwango T. A., 2017. La commercialisation des champignons comestibles au Cameroun. Revue Scientifique et Technique Forêt et Environnement du Bassin du Congo, 8 : 65-71.

Ebenye H. M. C., Taudière A., Niang N., Ndiaye C., Sauve M., Onguene N. A., et al., 2016. Ectomycorrhizal fungi are shared between seedlings and adults in a monodominant Gilbertiodendron dewevrei rain forest in Cameroon. Biotropica, 49 (2): 256-267.

Egbe E. A., Tonjock R. K., Ebai M. T., Nji T., Afui M. M., 2013. Diversity and distribution of macrofungi (mushrooms) in the Mount Cameroon region. Journal of Ecology and the Natural Environment, 5 (10): 318-334.

Eyi N. H., Degreef J., De Kesel A., 2011. Champignons comestibles des forêts denses d'Afrique centrale. Taxonomie et identification. Abc Taxa, 10, 254 p.

Eyi Ndong H. C., Mounguengui S., Attéké C., Ndong G. O., 2014. Variation of the Consumption of Mushrooms by Pygmies and Bantus in the North of Gabon. Advances in Microbiology, 4 (16): 1212-1221. http://dx.doi.org/10.4236/ aim.2014.416131

Fadeyi O. G., Badou S. A., Aignon H. L., Codjia J. E., Moutouama J. K., Yorou N. S., 2017. Études ethnomycologiques et identification des champignons sauvages comestibles les plus consommés dans la région des Monts-Kouffe au Bénin (Afrique de l'Ouest). Agronomie Africaine, 29 (1) : 93-109.

FAO, 2004. Non-wood forest products. Wild edible fungi: a global overview of their use and importance. Rome, Italy, FAO Publications. http://www.etre-bien.eu/ reishi-les-6-vertus-dun-champignon-fantastique/

Feeney M. J., Dwyer J., Hasler-Lewis C. M. E., et al., 2014. Mushrooms and Health Summit Proceedings. The Journal of Nutrition, 144: 1128S-1136S.

Fortin J. A., Plenchette C., Piché Y., 2008. Les mycorhizes : La nouvelle révolution verte. Montréal, Canada, Éditions Multimondes, $132 \mathrm{p}$.

Franqueville A., 1973. Atlas régional Sud-Ouest. République Unie du Cameroun. Yaoundé, Cameroun, Orstom.

Guissou K. M. L., Yorou N. S., Sankara P., Guinko S., 2015. Assessing the toxicity level of some useful mushrooms of Burkina Faso (West Africa). Journal of Applied Biosciences, 85: 7784-7793.

Hall I. R., Zambonelli A., Primavera F., 1998. Ectomycorrhizal Fungi with Edible Fruiting Bodies 3. Tuber magnatum, Tuberaceae. Economic Botany, 52 (2): 192-200.

Härkönen M., Saarimäki T., Mwasumbi L., 1994. Edible and poisonous mushrooms of Tanzania. African Journal of Mycology and Biotechnology, 2 (2): 99-123.

Härkönen M., Niemelä T., Mwasumbi L., 2003. Tanzanian mushrooms - Edible, harmful and other fungi. Norrlinia, 10: 1-200.

Heim R., 1942. Nouvelles études descriptives sur les Agarics termitophiles d'Afrique tropicale. Archives du Muséum National d'Histoire Naturelle, 6 (18) : 107-166. 
Hennings P., 1895. Fungi Camerunenses I. Botanische Jahrbücher für Systematik, 22: 72-111.

Johnsy G., Davidson S., Dinesh M. G., Kaviyarasan V., 2011. Nutritive value of edible wild mushrooms collected from the Western Ghats of Kanyakumari District. Botany Research International, 4 (4): 69-74. https://pdfs.semanticscholar. org/15ab/665a95d12d541fofe6ccc6161a18de9a932b.pdf

Kalač P., 2013. A review of chemical composition and nutritional value of wild-growing and cultivated mushrooms. Journal of the Science of Food and Agriculture, 93 (2): 20918. Doi: $10.1002 /$ jsfa.5960.

Kamou H., Nadjombe P., Gbogbo A. K., Yorou S. N., Batawila K., Akpagana K., Guelly K. A., 2017. Les champignons ecto-mycorrhiziens consommés par les Bassar et les Kabyè, peuples riverains du Parc National Fazao-Malfakassa (PNFM) au Togo (Afrique de l'Ouest). Revue Marocaine des Sciences Agronomiques et Vétérinaires, 5 (2) : 154-162.

Kouame K. B., Koko A. C., Diomande M., Konate I., Assidjo N. E., 2018. Caractérisation physicochimique de trois espèces de champignons sauvages comestibles couramment rencontrées dans la région du Haut-Sassandra (Côte d'Ivoire). Journal of Applied Biosciences, 121: 12110-12120.

Lãessoĕ T., Del Conte A., 2001. L'encyclopédie des champignons. Paris, France, Bordas, 256 p.

Letouzey R., Mouranche R., 1952. Ekop du Cameroun. Nogent-sur-Marne, France, Centre technique forestier tropical, Publication 4.

Lister C. E., 2015. Nutritional Analysis of Mushrooms - A summary. A Plant \& Food Research report prepared for Meadow Mushrooms Ltd, 22 p. https://www.meadowmushrooms. co.nz/media/1460/final-nutritional-analysis-of-meadowmushrooms-a-summary.pdf

Malaisse F., De Kesel A., N'Gasse G., Lognay G., 2008. Diversité des champignons consommés par les pygmées Bofi de la Lobaye (République centrafricaine). Géo-EcoTrop, 32: 1-8. http://www.geoecotrop.be/uploads/publications/pub 321 840454.pdf

Mueller G. M., Schmit J. P., Leakock P. R., Buyck B., et al., 2007. Global diversity and distribution of macrofungi. Biodiversity and Conservation, 16: 37-48. http://dx.doi. org/10.1007/s10531-006-9108-8

Newbery D. M., Alexander I. J., Thomas D. W., Gartlan J. S., 1988. Ectomycorrhizal rain-forest legumes and soil phosphorus in Korup National Park, Cameroon. New Phytologist, 109: 433-450.

Njouonkou A.-L., 2011. Taxonomie, systématique et étude phylogénétique des genres Lentinus Fr. et Pleurotus (Fr.) Kramer au Cameroun basée sur la morphologie et les séquences des régions ITS de l'ADNr et identification des enzymes extracellulaires oxydases de quelques champignons lignivores. Thèse de doctorat, Université de Yaoundé I, Cameroun, $198 \mathrm{p}$.

Njouonkou A. L., De Crop E., Mbenmoun A. M., Kinge T. R., Biyé E. H., Verbeken A., 2016. Diversity of edible and medicinal mushrooms used in the Noun Division of the West Region of Cameroon. International Journal of Medicinal Mushrooms, 18 (5): 387-396.

Oei P., 1996. Mushroom cultivation: with special emphasis on appropriate techniques for developing countries. Kerkwerve, The Netherlands, Backhuys Publishers, 284 p.
Olivry J.-C., 1986. Fleuves et rivières du Cameroun. Paris, France, MESRES/ORSTOM, coll. Monographies hydrologiques 9, 32 p. http://horizon.documentation.ird.fr/exl-doc/pleins textes/pleins textes 5/b fdi 12-13/16102.pdf

Onguene A. N., 2000. Diversity and dynamics of mycorrhizal association in tropical rain forest with different disturbance regimes in South Cameroun. PhD Thesis, Wageningen University, Tropenbos, The Netherlands, $167 \mathrm{p}$.

Onguene A. N., Kuyper T. W., 2001. Diversity and abundance of mycorrhizal association in the rain forest of South Cameroon. Tropenbos Cameroon Programme. Forest Ecology and Management. 140: 277-287.

Oso B. A., 1975. Mushrooms and the Yoruba people of Nigeria. Mycologia, 67 (2): 311-319.

Oso B. A., 1977. Mushrooms in Yoruba mythology and medicinal practices. Economic Botany, 31: 367-371.

Rammeloo J., Walleyn R., 1993. The edible fungi of Africa South of the Sahara. A literature survey. Scripta Botanica Belgica, 5: 1-62.

Saarimäki T., Härkönen M., Mwasumbi L., 1994. Tanzanian mushrooms and their uses 3. Termitomyces singidensis, sp. nov. Karstenia, 34: 13-20. http://www.funga.fi/Karstenia/ Karstenia\%2034-1\%201994-3.pdf

Teke N. A., Kinge T. R., Bechem E., Nji T. M., Ndam L. M., Mih A. M., 2018. Ethnomycological study in the Kilum-ljim mountain forest, Northwest Region, Cameroon. Journal of Ethnobiology and Ethnomedicine, 14: 25. https://doi. org/10.1186/s13002-018-0225-8

Tonjock R. K., Ebai M., Tabi Afui M. M., Egbe A. E., Njouonkou L., et al., 2013. Ethnomycological studies of edible and medicinal mushrooms in the Mount Cameroon region (Cameroon, Africa). International Journal of Medicinal Mushrooms, 13 (3): 299-305.

Tonjock R. K., Nkengmo A., Theobald M., Ache N. A., Afui M. M., 2017. Species Richness and Traditional Knowledge of Macrofungi (Mushrooms) in the Awing Forest Reserve and Communities, Northwest Region, Cameroon. Journal of Mycology, Article ID 2809239, 9 p. https://doi. org/10.1155/2017/2809239

Yorou S. N., De Kesel A., 2002. Connaissances ethnomycologiques des peuples Nagot du centre du Bénin (Afrique de l'Ouest). AETFAT Congress 16, Bruxelles, 2000. Systematics and Geography of Plants, 71: 627-637.

Bois et Forêts des Tropiques - Revue scientifique du Cirad 